\title{
Utilisation des composés phénoliques pour le marquage de pools géniques de Tournesol cultivé
}

\author{
Claire SANLAVILLE, Maurice JAY \& Joël GUIARD* \\ avec la collaboration technique de Patrick SALlaS* \& Liliane MOREAU* \\ Laboratoire de Biologie micromoléculaire et Phytochimie, Université Lyon I, 43, boulevard du II novembre \\ 1918, F 69622 Villeurbanne \\ * I.N.R.A., Groupe d'Etude et de Contrôle des Variétés et des Semences, Centre de Recherches de Versailles, \\ La Minière, F 78280 Guyancourt
}

RÉSUMÉ

\begin{abstract}
Le contenu phénolique foliaire de 75 individus représentatifs de 15 lignées de tournesol, appartenant à 5 pools géniques (ensembles de lignées de forte ressemblance et d'origine vraisemblablement commune) et mises en culture dans des conditions semblables, a été analysé par Chromatographie Liquide Haute Performance puis traité par Analyse en Composantes Principales. Les variations observées reposent uniquement sur des différences de proportions relatives entre composés phénoliques. Alors que les lignées d'un même pool génique sont similaires, des différences notables sont observées entre pools géniques. Ainsi, l'utilisation du seul critère phénolique pourrait être équivalente en pratique à celle de tout un ensemble de caractères morphologiques, physiologiques ou agronomiques sur lesquels est basée habituellement la reconnaissance variétale.
\end{abstract}

Mots clés additionnels : Flavonoïdes, acides phénols, Helianthus annuus.

Use of phenolic compounds as markers of cultivated sunflower gene pools.

The phenolic content of leaves from 75 individuals representative of 15 lines of sunflowers, themselves belonging to 5 gene pools (groups of lines highly similar and supposed to have a common origin) and cultivated under equivalent conditions, was analyzed by High Pressure Liquid Chromatography and treated by Principal Component Analysis. The variation observed concerned only different relative proportions of phenolic compounds. While lines belonging to the same gene pool were similar, noteworthy differences appeared between gene pools. Thus, analysis of phenolic compounds could alone be practically equivalent to use of the large sum of morphological, physiological and agronomic characters on which the recognition of lines is usually based.

Additional key words : Flavonoids, phenolic acids, Helianthus annuus.

\section{INTRODUCTION}

Le tournesol est une plante d'intérêt agronomique certain, comme le montre le développement de sa culture ces dernières années.

La commercialisation des semences est assujettie en France à l'inscription des variétés correspondantes au Catalogue Officiel. Cette inscription n'est accordée qu'à l'issue, entre autres, d'une épreuve "Distinctionnouveauté - Homogénéité - Stabilité » (D.H.S.) d'une durée de 2 à 3 ans, portant sur l'hybride ainsi que sur ses géniteurs. Cet examen, réalisé par le secteur «Variétés» du G.E.V.E.S. (Groupe d'Etude et de contrôle des Variétés Et des Semences) de l'I.N.R.A., fait actuellement appel uniquement à des caractères morphologiques, phénologiques et agronomiques des appareils végétatif et reproducteur. A l'expérience, il est parfois difficile, sur la base de ces seuls caractères, de distinguer une lignée d'une autre ou de son géniteur, mais il est tout aussi difficile de conclure de façon formelle à une non-distinction.

Parmi les critères chimiques pouvant permettre la distinction variétale, le polymorphisme isozymique n'a été que très peu employé chez le tournesol. Une dizaine de systèmes enzymatiques a fait l'objet d'investigations, concernant essentiellement leur déterminisme génétique (en particulier : TORRES, 1983 ; KALHER \& LAY, 1985). Mais leur utilisation comme marqueurs biochimiques de populations sauvages ou de lignées cultivées n'a pas donné lieu à publication, bien que des travaux soient actuellement en cours (TORRES, comm. pers., 1985 ; GRENECHE, comm. pers., 1987). 
Nous proposons, dans le contexte des critères chimiques, une approche micromoléculaire de la variabilité des lignées sélectionnées de tournesol, et plus précisément, l'utilisation des produits d'accumulation du métabolisme phénolique. L'originalité de ce métabolisme provient d'une part d'une forte diversité structurale par laquelle peut s'exprimer une large gamme de choix métaboliques, et d'autre part d'une forte sensibilité aux actions de sélection (ARDOUIN et al., 1985, 1987). Dans le cas particulier du tournesol, les 2 grandes classes de composés phénoliques, flavonoïdes (squelette de base $\mathrm{C15}$ ) et acides phénols (squelette de base $\mathrm{C} 7$ ou $\mathrm{C} 9$ ) ont fait l'objet d'investigations. Les composés flavoniques ont été étudiés essentiellernent chez les espèces sauvages à des fins taxinomiques (HARBORNE \& SMITH, 1978 ; HERZ \& DE GROOTE, 1977 ; MELEK et al., 1985 ; OHNO et al., 1981 ; SCHILLING \& MABRY, 1981 ; SCHILLING, 1983 ; WADDELL, 1973), tandis que les acides phénols l'ont été surtout chez le tournesol cultivé, sur les akènes, en tant que contaminants (LEUNG et al., 1981 ; MIKOLAJCZAK et al., 1970 ; ROBERTSON, 1975 ; SABIR et al., 1974), ou sur les organes foliaires ou caulinaires, en relation avec la phytopathologie (AvILA, 1984 ; BAZZALO et al., 1985 ; YANG, 1986). Cette apparente compartimentation des utilisations des composés phénoliques, C15 d'une part, et $C 7$ ou C9 d'autre part, relève d'un simple fait d'usage, les composés flavoniques, généralement plus stables, étant traditionnellement utilisés en chimiotaxinomie, tandis que les acides phénols se sont vus depuis longtemps attribuer un rôle dans l'interaction avec les pathogènes.

Nous voulions tenter de répondre aux questions suivantes :

- existe-t-il, chez le tournesol cultivé, une diversité phénolique suffisante pour permettre l'étude de la variabilité des lignées sélectionnées (entre lignées et au sein de chacune d'elles) ?

- quelle est l'organisation de cette variabilité phénolique? Plus précisément,

- deux individus se ressemblant d'après les critères retenus par le G.E.V.E.S. (par exemple 2 individus d'une même lignée, ou de 2 lignées d'un même pool génique) ont-ils une expression phénolique comparable?

- réciproquement, le critère phénolique est-il, à lui seul, capable de permettre la distinction entre pools géniques, que l'on ne peut différencier que par toute une somme de caractères morphologiques/phénologiques/agronomiques ?

Dans le but d'approcher au mieux la variabilité génotypique inter-lignées, nous avons placé les lignées dans des conditions aussi identiques que possible.

\section{MATÉRIEL ET MÉTHODES}

\section{A. Matériel végétal}

Nous avons étudié l'expression phénolique foliaire de 15 lignées simples, correspondant à 7 couples $A$ (mâles stériles) et $\mathbf{B}$ (mâles fertiles mainteneurs de stérilité), et à une lignée sous la seule forme A. Ces 15 lignées sont représentatives de 5 pools géniques, c'est-à-dire de 5 types morphologiques/physiologiques/agronomiques bien définis, et vraisemblablement d'origine commune. Ces lignées ont été testées par le G.E.V.E.S., qui a conclu à une très forte ressemblance entre lignées d'un même pool génique.

Ces 5 pools géniques, numérotés de 1 à 5 , sont constitués des lignées suivantes :

- 1: HA89A, 133A, 133B, 163A, 163B ;

$-2: 166 \mathrm{~A}, 166 \mathrm{~B}, 186 \mathrm{~A}, 186 \mathrm{~B}$;

- $3: 172 \mathrm{~A}, 172 \mathrm{~B}$

$-4: 176 \mathrm{~A}, 176 \mathrm{~B}$;

- $5: 181 \mathrm{~A}, 181 \mathrm{~B}$.

Ces lignées ont été mises simultanément en culture à La Minière (Yvelines) en plein champ en 1985.

Cinq individus par lignée ont fait l'objet d'un prélèvement à la floraison. Celui-ci a porté sur une feuille « jeune-adulte », c'est-à-dire juste arrivée à maturité physiologique et à complet développement (correspondant alors à la $6^{\mathrm{e}}$ ou $7^{\mathrm{e}}$ feuille à partir du capitule).

Le matériel végétal a été séché à l'air libre et à température ambiante.

\section{B. Analyse de l'expression phénolique}

\section{Préparation des extraits}

Chaque échantillon-feuille a été soumis individuellement aux opérations suivantes :

- extraction du bloc phénolique : 2 macérations successives ont été réalisées dans un mélange d'eau et d'alcool (40:60), destiné à solubiliser en particulier l'ensemble des composés phénoliques; les 2 macérats ont été réunis et concentrés à sec sous pression réduite à $40^{\circ} \mathrm{C}$;

- purification partielle du bloc phénolique : le résidu sec a été repris par de l'eau bouillante (afin d'éliminer en partie les composés chlorophylliens et terpéniques, très lipophiles), et extrait par de l'acétate d'éthyle (qui solubilise préférentiellement les composés phénoliques) ; ce dernier a été évaporé à sec et le résidu repris par un petit volume de méthanol, constituant l'extrait phénolique individuel, prêt à être analysé par chromatographie.

\section{Analyse chromatographique}

Celle-ci a été réalisée par Chromatographie Liquide Haute Performance (C.L.H.P.) sur colonne C18 (Microbondapak, $10 \mu, 300 \times 4 \mathrm{~mm}$ ), le système d'élution étant un gradient d'acétonitrile dans l'eau, en présence d'acide acétique, et la détection spectrophotométrique réalisée à $328 \mathrm{~nm}$ (longueur d'onde d'absorption maximale des extraits).

\section{Traitement des données}

Les profils C.L.H.P. ainsi obtenus ont été relevés par repérage des pics d'après leur temps de rétention. Les pics correspondant à des composés instables, donc non fiables, n'ont pas été pris en compte. Les pics conservés ont été quantifiés par leur hauteur, qui a été ensuite exprimée en p. 100 de la somme des hauteurs de pics du profil correspondant. Chaque pic représente un ou quelques composés très affines (des propriétés 
similaires de rétention sur la colonne s'expliquent en général par une structure chimique très proche).

Il est ensuite possible de tracer pour chaque extrait le profil simplifié par informatique (logiciel graphique, AUDA, 1983).

Les données ont été traitées par Analyse en Composantes Principales normée (ACP normée). Il n'a pas été réalisé d'Analyse Discriminante selon le facteur « lignée », le nombre d'individus par classes = lignées (5) étant faible par rapport au nombre de variables (21) et de classes (15), ni selon le facteur " pool génique ", l'échantillonnage étant très inégal d'un pool génique à l'autre.

\section{RÉSULTATS}

Il est possible de mesurer 21 pics suffisamment individualisés sur l'ensemble des échantillons, et correspondant à des composés stables.

La figure 1 présente les profils phénoliques foliaires simplifiés moyens par lignée (sur 5 individus). Les profils individuels ne sont pas rapportés ici, car ils sont très semblables pour une même lignée de sorte que le profil moyen correspondant suffit à refléter les capacités de la lignée.

La figure 2 montre les plans 1, 2 et 3, 4 des individus et les cercles de corrélations variables/facteurs correspondants du traitement par ACP normée des profils
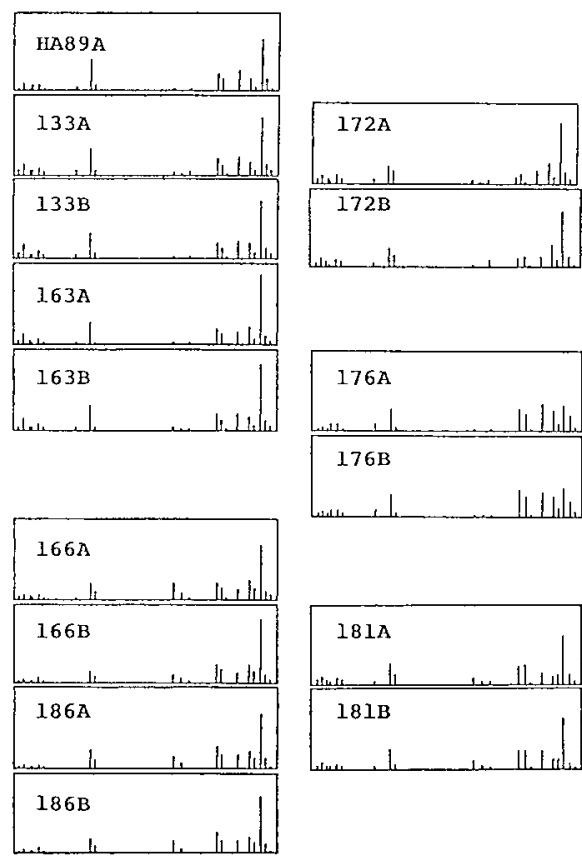

Figure 1

Profils phénoliques foliaires simplifiés moyens par lignée (sur 5 individus) de 15 lignées représentatives de 5 pools géniques de tournesol cultivé. Abscisse : temps de rétention des pics ; ordonnée : proportion de chacun de ces pics.

Simplified average (on 5 individuals) phenolic profiles of leaves from 15 lines representative of 5 gene pools of cultivated sunflower. $x$-axis : peak retention times, $y$-axis : relative ratio of each peak.
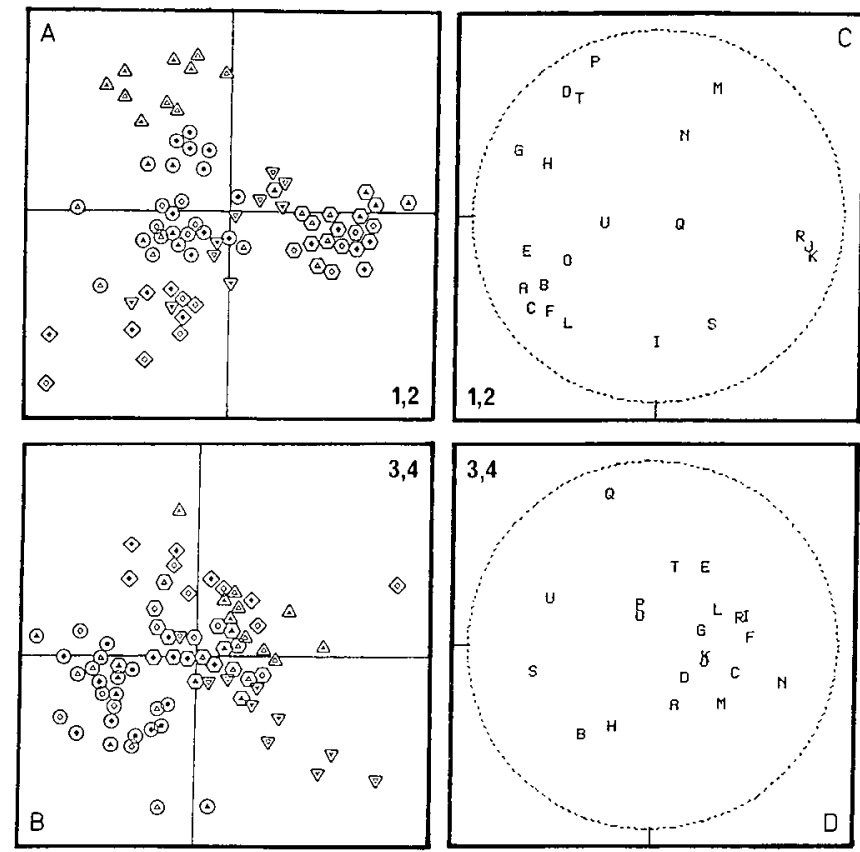
(- HA89A
(- 166A
(c) 133A
(0) $166 \mathrm{~B}$
(C) $133 \mathrm{~B}$
(4) $186 \mathrm{~A}$
(-) 163A
(4) $186 \mathrm{~B}$
(1) $163 \mathrm{~B}$

(-) 172A

$\triangle 176 \mathrm{~A}$

D 181A

사 172B

$\triangle 176 \mathrm{~B}$

V $181 B$

Figure 2

Dépouillement du traitement par Analyse en Composantes Principales Normée des profils phénoliques foliaires de 15 lignées 15 pools géniques): cartes factorielles des individus 1,2 (fig. A) et 3.4 (fig. B); cercles de corrélations variables/facteurs 1,2 (fig. C) et 3,4 (fig. D) correspondants.

$A, B, C \ldots T, U:$ pics.
Principal Component Analysis treatment of the phenolic profiles of leaves from 15 lines (5 gene pools) : distribution of the individuals on the F1 $\times F 2$ (fig. A) and F3 $\times F 4$ (fig. B) planes; corresponding variables/factors correlation circles $F 1 \times F 2$ (fig. C) and $F 3 \times F 4$ (fig. $D$ ).

$A, B, C \ldots T, U ;$ peaks. 
phénoliques foliaires de l'échantillonnage. Les pourcentages d'inertie de ces axes sont, respectivement, $29,7 \%, 21,4 \%, 14,5 \%$ et $9,6 \%$, soit au total $75,2 \%$ de la variabilité totale.

\section{DISCUSSION}

\section{A. Nature de la variation phénolique}

Les extraits foliaires diffèrent entre eux d'un point de vue non pas qualitatif, mais uniquement semiquantitatif : les variations entre profils moyens que l'on peut observer sur la figure 1 ne reposent que sur des différences de proportions relatives entre pics. Ainsi, la variabilité phénolique du tournesol cultivé, mesurée ici toutes conditions égales par ailleurs, provient de processus différentiels de régulation des diverses voies du métabolisme phénolique.

\section{B. Variation intra-lignées}

Les lignées étudiées apparaissent, d'une façon générale, assez homogènes du point de vue de leur expression phénolique (comme le confirme la distribution des points-individus sur la fig. 2). Ainsi, les individus provenant du même site de sélection et donc résultant du même tri de sélection ont un métabolisme phénolique comparable, ce qui semble impliquer que la sélection stabilisatrice de caractères d'intérêt agronomique se répercute aussi sur le métabolisme phénolique par son homogénéisation concomitante.

\section{Variation intra-pools géniques}

Les lignées d'un pool génique donné ont une expression phénolique comparable (fig. 1 et 2). Il apparaît d'ailleurs sur la figure 2 que la variation inter-lignées intra-pools géniques est du même ordre de grandeur que la variation inter-individus intra-lignées car la dispersion des points-individus correspondants est comparable dans les 2 cas :

- les représentants $A$ et $B$ d'un même couple ne nous semblent pas différents - bien que leurs nuages de points-individus respectifs soient légèrement décalés. L'expression phénolique ne paraît donc pas pouvoir marquer le caractère stérilité/fertilité mâle ;

- les lignées de couples (A, B) différents mais d'un même pool génique sont similaires.

Ainsi, nous constatons que des processus de sélection comparables, aboutissant à des lignées équivalentes du point de vue des caractères jugés par le G.E.V.E.S., conduisent aussi à des produits de sélection qui nous paraissent semblables du point de vue de leur expression phénolique.

\section{Variation inter-pools géniques}

D'un pool génique à l'autre, par contre, des différences notables d'expression phénolique peuvent être observées, par l'examen des profils simplifiés (fig. 1) ou de leur traitement multivarié (fig. 2). Les principales caractéristiques phénoliques de chaque pool géni- que, révélées par l'ACP normée, sont résumées dans le tableau 1. Ainsi, des lignées dissemblables entre elles d'après les critères retenus par le G.E.V.E.S. présentent par ailleurs des tendances métaboliques différentes, et peuvent donc être séparées par l'analyse de leur contenu phénolique. Des pressions de sélection différentes, aboutissant à des types bien nets selon le G.E.V.E.S., conduisent ainsi également à des équilibres phénoliques distincts.

\section{TABLEAU 1}

Principales caractéristiques phénoliques des 15 lignées (5 pools géniques) analysées.

+ ou - : proportions plus forte ou moins forle que chez les autres lignées (d'après les fig. 1 et 2).

Main phenolic characteristics of the 15 lines (5 gene pools) analyzed. + or - : proportions higher or lower than in the other lines (according to fig. 1 and 2).

\begin{tabular}{|c|c|c|c|c|c|}
\hline \multirow{4}{*}{ Lignées } & \multicolumn{5}{|l|}{ НА 89 А } \\
\hline & $133 \mathrm{~A}$ & $166 \mathrm{~A}$ & & & \\
\hline & 133B & $166 \mathrm{~B}$ & & & \\
\hline & $163 \mathrm{~A}$ & $186 \mathrm{~A}$ & $172 \mathrm{~A}$ & $176 \mathrm{~A}$ & $181 \mathrm{~A}$ \\
\hline Pics & $163 \mathrm{~B}$ & $186 \mathrm{~B}$ & $172 \mathrm{~B}$ & $176 \mathrm{~B}$ & $181 \mathrm{~B}$ \\
\hline A & & - & + & & \\
\hline B & + & - & + & & \\
\hline $\mathrm{C}$ & & - & + & & \\
\hline $\mathrm{D}$ & & & & + & \\
\hline $\mathrm{E}$ & & - & + & & \\
\hline $\mathrm{F}$ & & - & + & & \\
\hline $\mathrm{G}$ & & - & & & \\
\hline $\mathrm{H}$ & + & - & & & \\
\hline I & & & + & & \\
\hline $\mathbf{J}$ & & + & & & \\
\hline $\mathrm{K}$ & & + & & & \\
\hline $\mathrm{L}$ & & & + & & \\
\hline M & & & - & + & \\
\hline $\mathrm{N}$ & & & - & + & + \\
\hline $\mathrm{O}$ & & & & & \\
\hline $\mathrm{P}$ & & & & + & \\
\hline$Q$ & & & & & - \\
\hline $\mathrm{R}$ & & + & & & \\
\hline $\mathrm{S}$ & + & & + & - & \\
\hline$T$ & & & & + & \\
\hline $\mathrm{U}$ & & & & & \\
\hline
\end{tabular}

\section{CONCLUSION}

Sur la base du seul critère phénolique, il est donc possible d'établir des ressemblances et des dissemblances entre lignées qui correspondent tout-à-fait à ce que révèle l'examen d'une somme de caractères morphologiques/phénologiques/agronomiques. La lecture de l'expression du métabolisme phénolique semble donc pouvoir être une méthode équivalente à l'examen complexe et global actuellement pratiqué.

Il est de notre intention de dépasser le simple marquage de pools géniques pour atteindre celui de caractères sélectionnés, var une étude de la corrélation entre expression phénolique et caractères physiologiques et surtout agronomiques. Le bien-fondé d'une telle démarche repose sur quelques travaux récents : AvILA (1984), BAZZALO et al. (1985) et YANG (1986) mettent par exemple en évidence l'intervention directe de certaines molécules phénoliques dans l'interaction hôtepathogène, ce qui pourrait permettre un marquage de résistances ou tolérances, moyennant une étude de la 
corrélation entre profils de la lignée en l'absence d'infection et degré de sensibilité lors de celle-ci.

Reçu le 20 mars 1987. Accepté le 4 février 1988.

\section{REMERCIEMENTS}

Nous exprimons nos vifs remerciements à MM. A. L. Roux et D. CHESSEL, Ecologie des eaux douces, UA CNRS 367, Université Lyon I, pour avoir mis à notre disposition les moyens de réaliser le traitement statistique de nos données.

\section{RÉFÉRENCES BIBLIOGRAPHIQUES}

Ardouin P., Fiasson J. L., Jay M., Lumaret R., Hubac J. M., 1985. Chemical diversification within the Dactylis glomerata L. polyploid complex (Gramineae), p. 3-15. In P. JACQuard, J. Heim \& J. AntoNovics. Genetic Differentiation and Dispersal in Plants. SpringerVerlag, Berlin, $448 \mathrm{p}$.

Ardouin P., Jay M., Lumaret R., 1987. Etude d'une situation de sympatrie entre diploïdes et tétraploïdes de Dactylis glomerata (Gramineae), sur la base du polymorphisme enzymatique et phénolique. Can. J. Bot., 65, 526-531.

Auda Y., 1983. Rôle des méthodes graphiques en analyse des données. Application au dépouillement des enquêtes écologiques. Thèse Doct. $3^{\mathrm{e}}$ cycle, Lyon, $127 \mathrm{p}$.

Avila F. J., 1984. The role of phenolic compounds in the resistance of sunflower to Sclerotinia sclerotiorum. Ph. D., Iowa, $108 \mathrm{p}$.

Bazzalo M. E., Heber E. M., Del Pero Martinez M. A., Caso O. H., 1985. Phenolic compounds in stems of sunflower plants inoculated with Sclerotinia sclerotiorum and their inhibitory effect on the fungus. Phytopathol. Z., 112, 322-332.

Harborne J. B., Smith D. M., 1978. Anthochlors and other flavonoids as honey guides in the Compositae. Biochem. Syst. Ecol., 6, 287-291.

Herz W., De Groote R., 1977. Desacetyleupaserrin and nevadensin from Helianthus pumilus. Phytochemistry, 16, 1307-1308

Kahler A. L., Lay C. L.. 1985. Genetics of electrophoretic variants in the annual sunflower. J. Hered., 76, 335-340.
Leung J., Fenton T. W., Clandinin D. R., 1981. Phenolic components of sunflower flour. J. Food Sci., 46, 1386-1388; 1393.

Melek F. R., Ahmed A. A., Mabry T. J., 1985. 6-Methoxylated flavones from Helianthus hirsutus and $H$. gracilentus. Rev. Latinoam. Quim., 16, 27.

Mikolajczak K. L., Smith C. R., Wolff I. A., 1970. Phenolic and sugar components of Armavirec variety sunflower (Helianthus annuus) seed meal. J. Agric. Food Chem., 18, 27-32.

Ohno N., Gershenzon J., Neuman P., Mabry T. J., 1981. Diterpene carboxylic acids and a heliangolide from Helianthus angustifolius. Phytochemistry, 20, 2393-2396.

Robertson J. A., 1975. Use of sunflower seed in food products. CRC Crit. Rev. Food Sci. Nutr., 6, 201-240.

Sabir M. A., Sosulski R. W., Kernan J. A., 1974. Phenolic constituents in sunflower flour. Agric. Food Chem., 22, 572-574.

Schilling E. E., 1983. Flavonoids in Helianthus series Angustifolii. Biochem. Syst. Ecol., 11, 341-344.

Schilling E. E., Mabry T. J., 1981. Flavonoids of Helianthus series Corona-Solis. Biochem. Syst. Ecol., 9, 161-163.

Torres A. M., 1983. Sunflowers (Helianthus annuus L.), p. 329-338. In S. D. TANKSLEY \& T. J. ORTON. Isozymes in Plant Genetics and Breeding, Part. B. Elsevier Science Publisher B. V., Amsterdam.

Waddell T. J., 1973. Hymenoxin : a flavone from Helianthus angustifolius. Phvtochemistry, 12, 2061.

Yang Z., 1986. Induced antifungal compounds in Helianthus annuus L. Ph. D., lowa, $75 \mathrm{p}$. 